\title{
Study of impurity behaviour during JET radiative boundary experiments
}

\author{
H Chen ${ }^{1,5}$, R Giannella ${ }^{2}$, N C Hawkes ${ }^{3}$, L Lauro-Taroni ${ }^{4}$, N J Peacock ${ }^{3}$ \\ and $M$ von Hellermann \\ JET Joint Undertaking, Abingdon, Oxon OX14 3EA, UK \\ ${ }^{1}$ Imperial College, The Blackett Laboratory, London SW7 2BZ, UK \\ ${ }^{3}$ EURATOM/UKAEA Fusion Association, Culham, Abingdon, Oxon OX14 3DB, UK \\ 2 CEA Cadarache, F-13108 St. Paul-lez-Durance, France \\ ${ }^{4}$ LCMM, Department of Meteorology, University of Reading, Reading RG6 6BB, UK
}

Received 30 May 2000, in final form 22 September 2000

\begin{abstract}
The transport of light impurity ions is investigated following neon and nitrogen gas puffing in JET ELMy H-mode. Upon achieving consistency among various ion radiation diagnostics through numerical simulations, the experimental ion transport coefficients are compared with the predictions of neoclassical theory at different regions of the plasma. The convection dominates the transport and, in the core, the transport coefficients approach the neoclassical value.
\end{abstract}

\section{Introduction}

The plasma-wall interaction has proved to be an outstanding challenge in the progress towards the next step fusion device $[1,2]$ because of the thermal load and erosion of the wall materials. One potential scenario for its solution relies on impurity radiation in the plasma boundary. The radiation from impurities could distribute the exhaust power onto a large area of the vessel walls, thereby avoiding high thermal loads and the dangerous erosion of the divertor targets and walls and suppressing the impurity influxes due to material erosion. By choosing the injecting impurity species, the radiation location can be optimized. A potential use of impurity injection is to apply a feedback to control the plasma performance in a future reactor.

The impurities employed to exhaust power by radiation in the plasma boundary may also penetrate the bulk plasma, leading to fuel dilution and strong radiation loss. In this paper the behaviour of the puffed radiators and intrinsic impurities in the JET bulk plasma is studied, focusing on the impurity particle transport, which allows the radiation profiles and the contamination of the bulk plasma during gas puffing experiments to be determined. The aim of this work is to study, quantitatively, in the bulk plasma, the puffed impurities that are used to establish a cold boundary in new JET configurations.

This work has used data from many JET diagnostics. Quantitative active charge exchange spectroscopy (CXS) [3] provides the light impurity density profiles, which make it possible

5 Present address: University of California, Lawrence Livermore National Laboratory, Livermore, CA 94551, USA. 


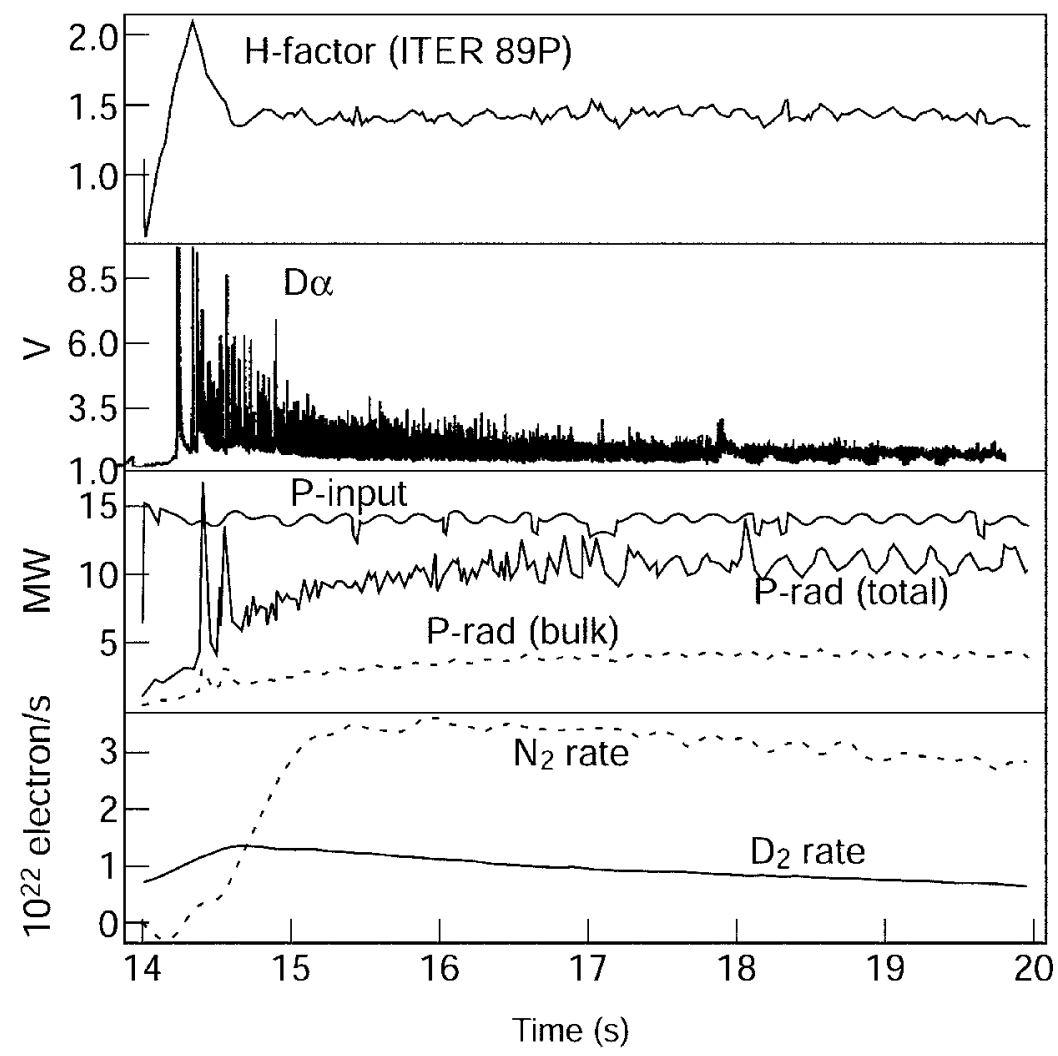

Figure 1. An overview of a nitrogen puffing discharge, pulse 33204.

to deduce the particle transport; $\mathrm{x}$-ray [4], XUV and VUV [5] spectrometers provide line intensities of passive emission from different ionization stages of the various impurities. The bolometry diagnostic [6] enables the total radiation from different regions of the plasma to be measured, while Abel inversion or tomographic reconstruction give the radial profile of the radiation.

The 1.5-dimensional (1.5D) predictive transport code SANCO [7-10] is used in this work to simulate the injected and intrinsic impurity transport and to study the consistency among data from different diagnostics. This code takes the main plasma parameters and a specified plasma geometry as inputs to calculate the non-coronal ionization balance by using the atomic data from ADAS [10] and model profiles of the impurity diffusion $D$ and convective velocity $V$. In the model, the $D$ and $V$ profiles are varied to achieve the best fit of the data. Consistency between the simulated and experimental data of independent diagnostics enables the validity of the model profiles of the $D$ and $V$ to be tested.

\section{The gas puffing experiment}

The nitrogen and neon gas puffing discharges analysed in this work are heated by $10-18 \mathrm{MW}$ of neutral beam injection. The plasma current is in the range 2.5-3.1 MA and a toroidal magnetic field of 2.4-2.8 $\mathrm{T}$ is used. These $\mathrm{H}$-mode discharges are characterized by a continuous small ELM activity and their energy confinement enhancement factors (H-factors) with respect to the 


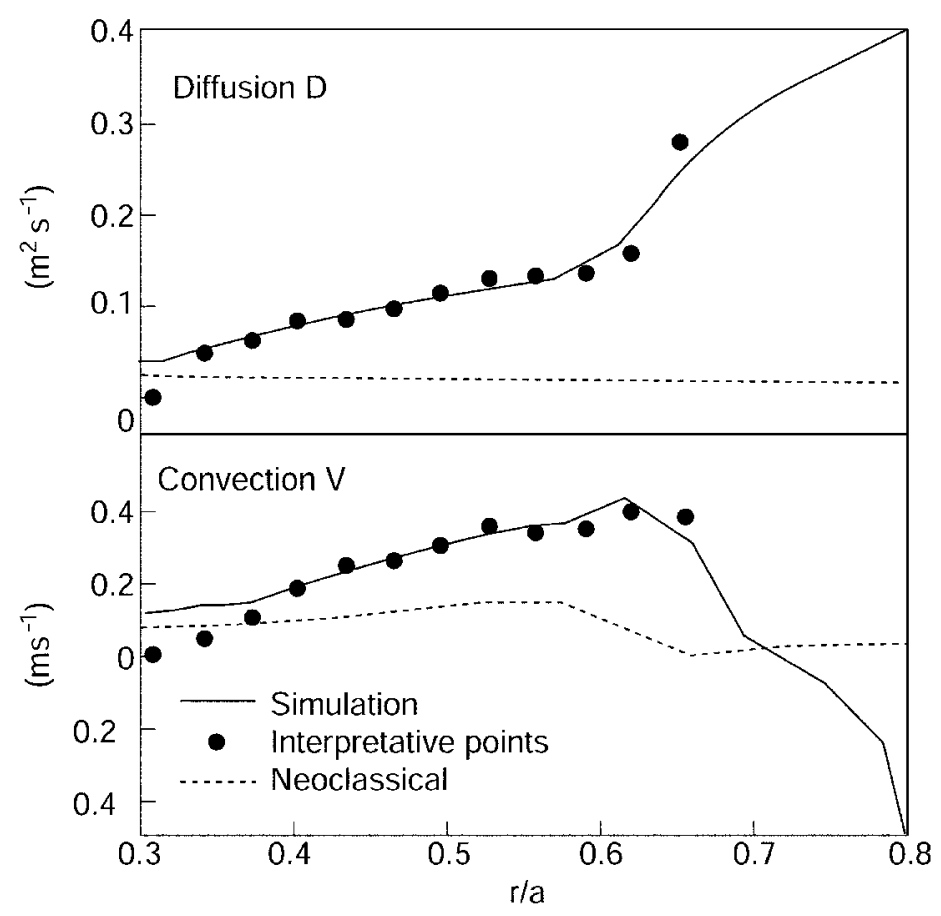

Figure 2. Neon $D(r)$ and $V(r)$ profiles obtained by the interpretative transport analysis and the predictive approaches using the SANCO code in discharge 32778.

ITER-89P scaling law [11] are about 1.5-2. The central electron temperature was $4-5 \mathrm{keV}$, and the electron density was $(6.5-8) \times 10^{19} \mathrm{~m}^{-3}$. In the bulk plasma region $(0<r / a<0.7)$, the temperature and density profiles were nearly flat, and the variation of the electron temperature and density profiles were less than $20 \%$. The electron temperature and density decrease quickly at the edge region $(r / a>0.8)$ to about $0.5 \mathrm{keV}$ and $1.5 \times 10^{19} \mathrm{~m}^{-3}$ at $r / a=0.95$, respectively. The line averaged effective charge $Z_{\text {eff }}$ in these discharges were in the range of 3.2-4.5. The neon gas was pulse puffed and the time duration was about $200 \mathrm{~ms}$. The neon density was about $1-2 \times 10^{17} \mathrm{~m}^{-3}$. In a strong gas puffed discharge, such as with a strong nitrogen puffing rate $\left(\sim 3.5 \times 10^{22}\right.$ electrons $\left.\mathrm{s}^{-1}\right)$, the $\mathrm{N}$ density in the plasma was in about $0.5-3 \times 10^{18} \mathrm{~m}^{-3}$. This results in a high total radiated power ( $80 \%$ of the total input power in pulse 33204$)$, as shown in figure 1. About one-third of the radiated power $(\sim 4 \mathrm{MW})$ is from the bulk plasma and the rest is from the divertor region. A lower radiation fraction $(\sim 40 \%$ of the total input power) was obtained in neon puffing discharges.

\section{Impurity transport}

We used the code SANCO to simulate the transport for the puffed impurities. This code has been described in detail elsewhere [7-9]. Briefly, for impurity at ionization state Z, its density can be described as a continuity equation:

$\frac{\partial n_{z}}{\partial t}=-\nabla \cdot\left(-D \vec{\nabla} n_{z}+V n_{z}\right)-\frac{n_{z}}{\tau_{\mathrm{p}}}-\left(S_{z} n_{z}+\alpha_{z} n_{z}\right)+\left(S_{z-1} n_{z-1}+\alpha_{z+1} n_{z+1}\right)$

The first term on the right-hand side is the divergence of the radial flux. The second term represents the parallel loss of particles: $n_{z} / \tau_{\mathrm{p}}$, where $\tau_{\mathrm{p}}$ is the particle confinement time in the 


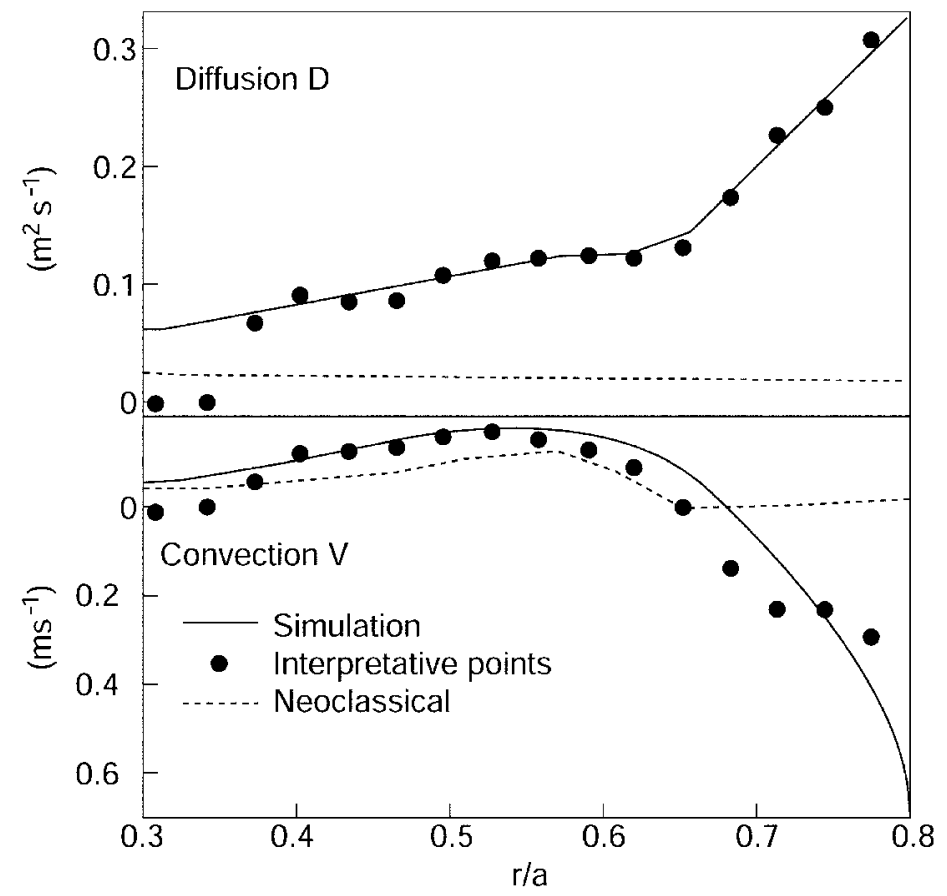

Figure 3. Carbon $D(r)$ and $V(r)$ profiles obtained by the interpretative transport analysis and the predictive approaches using the SANCO code in discharge 32778.

plasma scrape-off layer (SOL). The remaining terms on the right-hand side of the equation account for the impurity ion atomic processes, where $S$ is the ionization rate coefficient and $\alpha$ the recombination coefficient. The first bracket is the loss to adjacent ionization stages by both ionization and recombination processes, and the second bracket is the source by the like processes from adjacent ionization stages. The boundary conditions are those in the plasma centre, where the impurity density gradient is zero. At the plasma boundary, $\Gamma_{\text {influx }}^{\psi=1}=n_{0}^{\psi=1} V_{0}=\Gamma_{\text {external }}+R_{\text {recyc }} \sum_{z} \Gamma_{\text {out-flux }}^{\psi=1}$ where $n_{0}$ and $V_{0}$ are the total density and velocity of the neutral particles entering the plasma. The flux coordinate $\psi=1$ indicates the boundary of the plasma. This total flux is set equal to the sum of an external flux, $\Gamma_{\text {external }}$, (representing, for example, gas fuelling) and a recycling flux density. For non-recycling impurities, such as laser-injected metallic impurities, it is assumed that the recycling factor $=0$.

In the simulation, a delta function was used to simulate the pulse impurity injection, such as neon puffing, in discharge 32778. For long injections such as N puffing in discharge 33204, the impurity influx was assumed following the profile of the gas puffing rate. For intrinsic impurity carbon, the influx function was assumed such that it can best represent the time history of low-charge $\mathrm{C}$ line emission, since low-charged $\mathrm{C}$ lines exist mostly at the very edge of the plasma and their population closely reflect the influx. Accurate recycling factors for these recycling impurities were unknown. To find a best approximation, we tried a range of recycling factors $\left(R_{\text {recyc }}=0.1-1\right)$, and used the value that could best reproduce the line emission of the low-charged impurity ions. As stated above, these low-charged ions could only exist at the edge given the plasma condition, and therefore provided a close reflection of the flux in the edge region. 


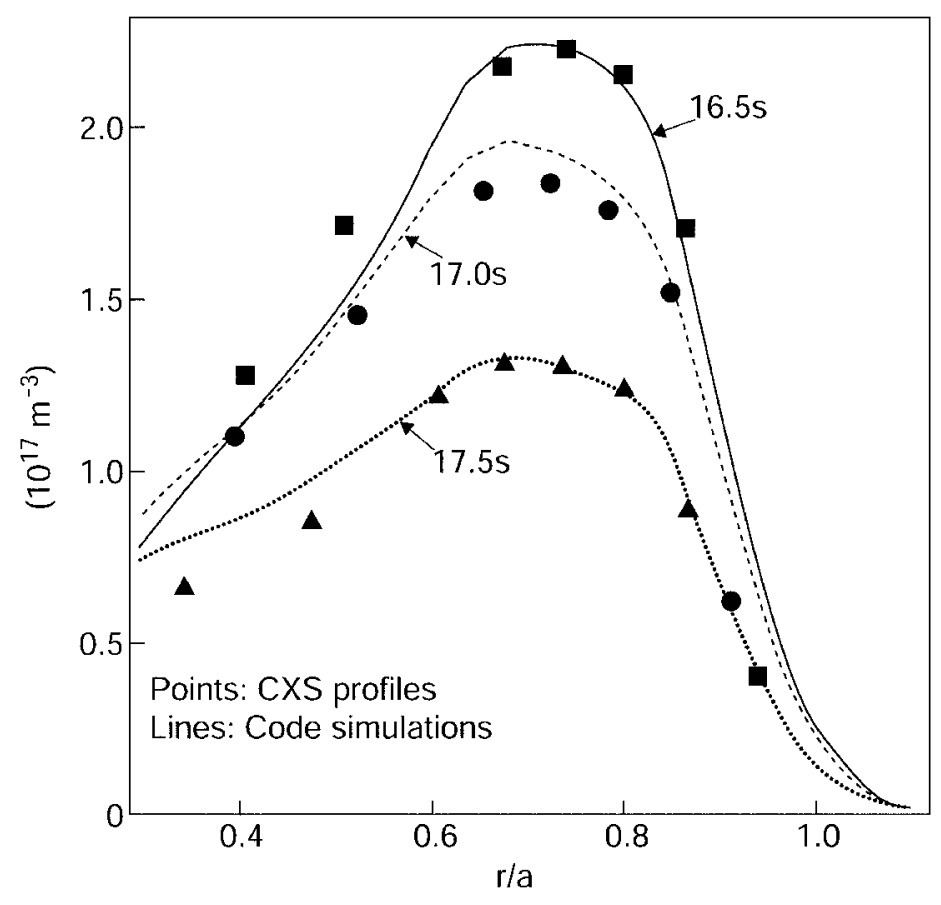

Figure 4. The fully stripped neon density profiles both from the charge exchange experiment and from code simulations at various times (pulse 32778).

Two approaches can be used to obtain the diffusion coefficient $D$ and convection velocity $V$. One, the 'predictive' method, is to insert trial profiles for $D$ and $V$ into the simulation code and then adjust these profiles until a good agreement is achieved between the simulation and the experimental data. The starting profiles of $D$ and $V$ in our analysis used the input from the 'interpretative' method, as described in the following paragraph. If the input from the 'interpretative' method is not available, the transport results from previously studied JET discharges can be used as the starting profiles of $D$ and $V$ in the predictive transport analysis. A successful model for impurity transport will predict the line radiation and the measurements from soft x-ray cameras and bolometers. For light impurities, such as neon, the H-like and He-like ions are found largely inside the separatrix in the JET tokamak, although close to the plasma edge. The time evolution of their line intensities, however, is of limited use for a determination of the transport parameter profiles because of their limited radial extent. Measurements of the impurity density profiles from CXS provide the necessary spatial information when applying this approach to the light impurity elements.

Another complementary approach, the 'interpretative' method [8,9], is based on the analysis of the functional relation between the normalized impurity fluxes and the gradients of the impurity densities that gives directly the diffusion coefficient and the convection velocity. At each radial position $r$ of the source-free region, the local ion flux $\Gamma_{I}$ can be derived from the continuity equation $\partial n_{\mathrm{I}} / \partial t=-(1 / r) \partial\left(r \Gamma_{\mathrm{I}}\right) / \partial r$. A linear fit to the data points in the transport model $\Gamma_{\mathrm{I}}=D \partial n_{\mathrm{I}} / \partial r+V n_{\mathrm{I}}$ gives the transport coefficients $D$ and $V$. This method is useful in that it provides a quantitative estimate of the uncertainties of the deduced transport parameters. Also, it is 'impartial' towards $D$ and $V$, and gives a good starting point for the predictive method. 


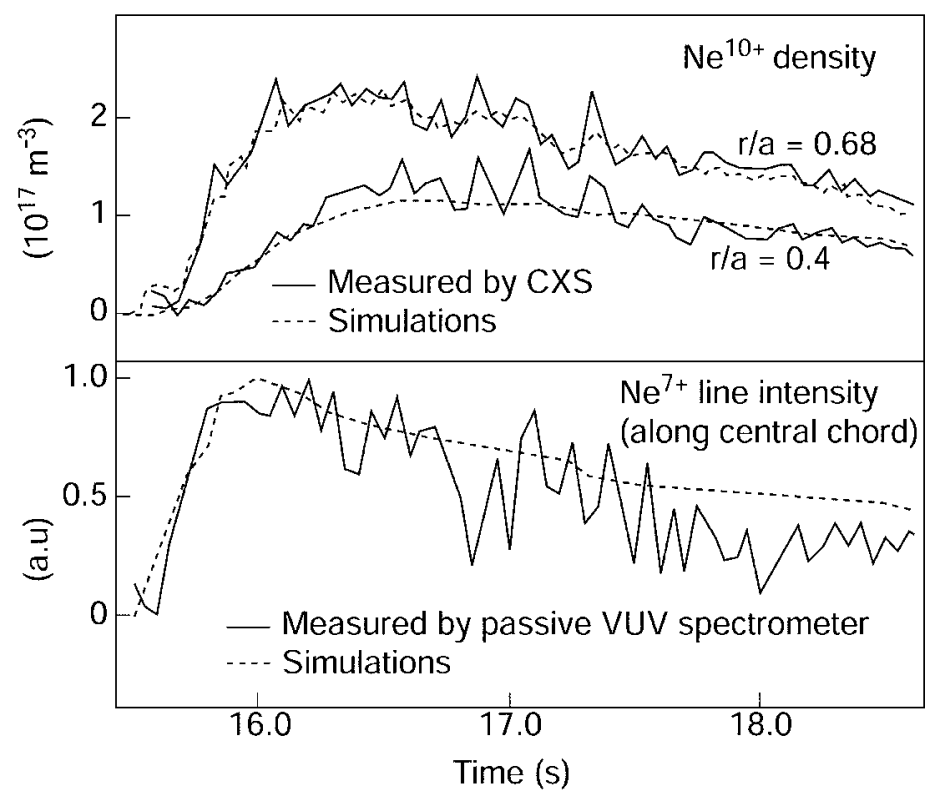

Figure 5. A good agreement between measurements and simulations for fully stripped neon (at two radii) (upper) and the line intensities of Li-like neon ions (lower) (pulse 32778).

A typical ELMy H-mode neon puffing discharge, pulse 32778, was studied in detail and both passive and active spectroscopy data were available for the intrinsic carbon and the injected neon. A $200 \mathrm{~ms}$ neon gas puff was injected into this discharge. Both transport analysis approaches were performed, with the SANCO code used to model the neon in these discharges. The resulting $D$ and $V$ profiles and their neoclassical transport predictions are shown in figure 2, while the corresponding results for carbon are shown in figure 3 . In the edge region $(r / a>0.8)$ the ion transport was strongly affected by the ELM activity, and it was not possible to derive the transport coefficients using the interpretative method due to the fluctuations in the experimental data. $D$ and $V$ were kept constant across the edge region. The simulations were optimized by comparing the results with the CXS ion density profiles and time histories of the line-of-sight integrated line emission from passive spectroscopy. It can be seen that the transport parameters, $D$ and $V$ profiles, derived from both the predictive and interpretative approaches are similar, particularly so in the case of carbon. The differences in the neon diffusion coefficients give some indication of the range of values expected from the simulations.

Little difference is observed between these coefficients obtained for the intrinsic impurity carbon $(Z=6)$ and the injected neon $(Z=10)$. This probably is due to the closeness of their atomic numbers, even though one is an intrinsic impurity and the other is an injected impurity. The discrepancy between the simulated transport coefficients and their neo-classical predictions decreases towards the plasma centre and it is noted that a similar conclusion was drawn for JET 'pellet enhanced pulses' (PEP) [12].

Both fully stripped $\mathrm{Ne}$ and $\mathrm{C}$ density profiles measured by active CXS are hollow, thus confirming that the impurities do not accumulate on-axis. To simulate these density profiles, positive (outwards) convection velocities inside $r / a<0.7$ and negative (inwards) outside $r / a>0.8$ are needed together with a low diffusion coefficient $\left(D=0.1-0.3 \mathrm{~m}^{2} \mathrm{~s}^{-1}\right)$. Transport parameters from both approaches are used to obtain the transport coefficients in the 


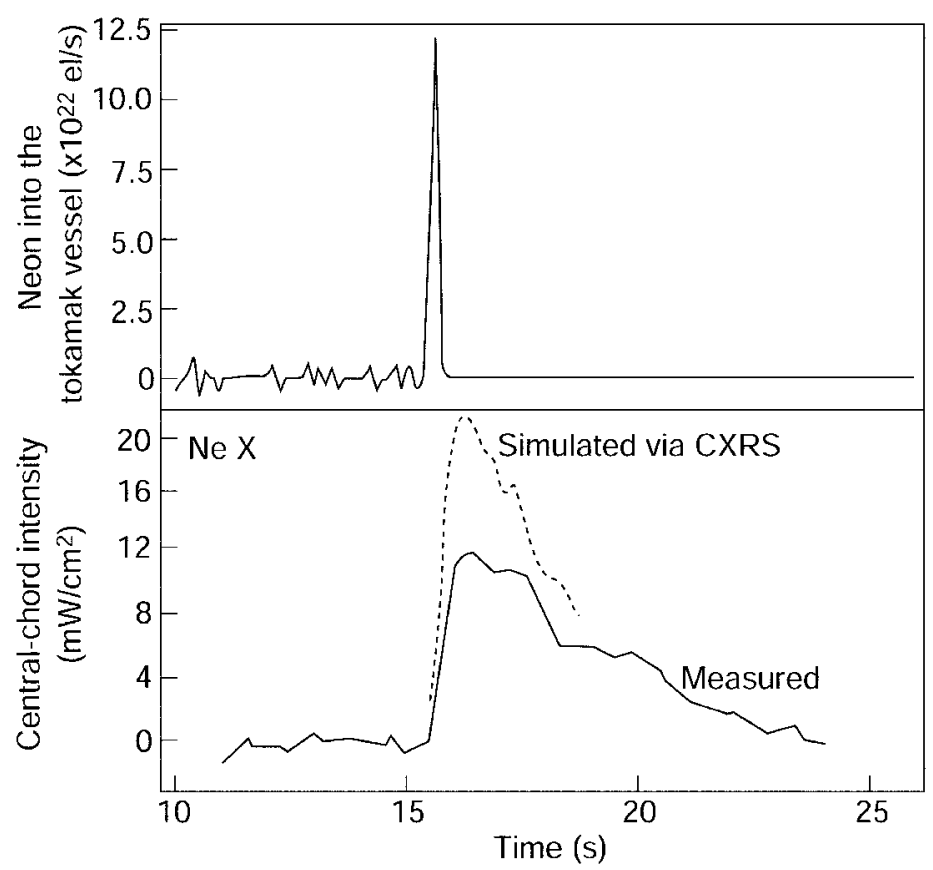

Figure 6. The results of simulations in pulse 32778 are compared with the absolute measurement of the $\mathrm{Ly}_{\beta}$ intensity.

simulations. It should be noted that for these impurities neoclassical transport theory predicts outwards convection velocities, as shown in figures 2 and 3. These transport parameters have reproduced well the experimental data and, as an example, the simulated neon density profiles at various times are compared with the CXS measurements in figure 4.

The time history of the line integrated emission from Li-like neon ions (from the VUV spectrometer) and the fully stripped neon densities (from the CXS measurement) are compared with the simulation results in figure 5. Whilst the CXS measurements yield the absolute value of the impurity density, allowing a full comparison with the simulation results, the VUV spectrometer is not calibrated and so only the normalized shapes of the line intensity can be compared. The fluctuations in the signal seen on the Li-like trace are due to ELMs.

In the nitrogen puffing discharges, the fully stripped nitrogen density profiles were not available (due to the complexity of the spectra near the nitrogen lines), and there was only a single measurement at $r / a=0.4$. The derived $\mathrm{C}(Z=6) D$ and $V$ profiles were used in the $\mathrm{N}(Z=7)$ simulation. These transport coefficients reproduced the time history of the total radiation and $\mathrm{H}$-like line brightness radiation. This further confirms that the light impurities, $\mathrm{Ne}, \mathrm{C}$ and $\mathrm{N}$, have similar transport parameters.

\section{The consistency of the quantitative impurity measurements}

To deduce the impurity density from the absolutely calibrated impurity line brightness measured by the passive spectrometers requires the impurity ionization balance from a non-coronal simulation code. Adjusting the simulated line brightness to the experimental measurements allows the impurities' (gas-puffed neon and intrinsic carbon) ion density profiles to be determined. At the same time, the ion density profiles of the same impurities can be 


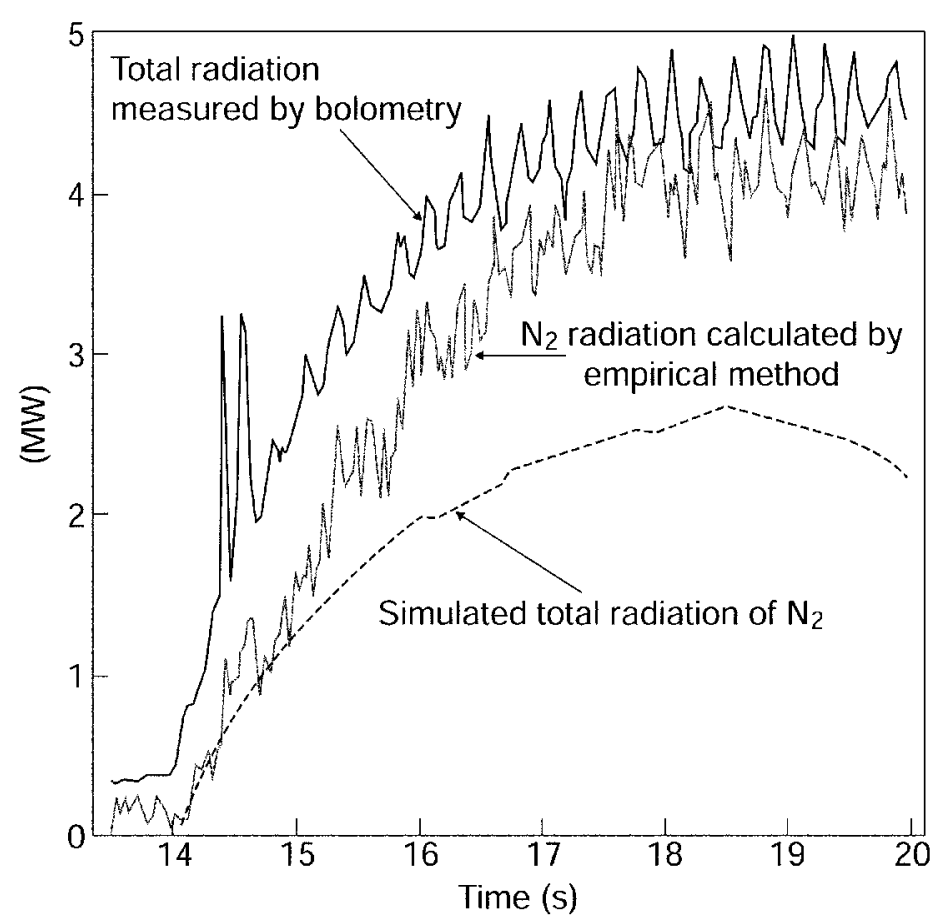

Figure 7. Simulated total radiation from $\mathrm{N}$ following closely the time evolution of the total radiation from the bulk plasma measured by bolometry. There is agreement in the absolute measurement to within a factor of two to three. (Pulse 33204, with $\mathrm{N}_{2}$ puffing.)

measured from active CXS. It is useful to compare the results from these two diagnostics quantitatively.

Comparisons between the results from the absolutely calibrated $\mathrm{x}$-ray spectrometer and CXS measurements were performed using numerical simulations. $\mathrm{Ly}_{\beta}$ was used in the simulation because the measurements are of a better quality than these of the $\mathrm{Ly}_{\alpha}$ line. The $\mathrm{H}$-like line brightness is chosen for this comparison, since it has the innermost emission shell and is located deeper inside the plasma where the uncertainties of the ion transport are lower. In the simulation, the impurity density was scaled to match the measured value from CXS at the time right after the injection. The calculated value of the line intensity of the H-like neon $\mathrm{Ly}_{\beta}$ was then compared to the calibrated measurement from the spectrometer. The results of simulations are compared with the absolute measurement of the $\operatorname{Ly}_{\beta}$ intensity in figure 6 . There is agreement to within a factor of two, which is good considering the uncertainty involved in the instrument calibration [13], the uncertainty in the CXS measurements and the uncertainty in the modelling. This analysis gives a point of reference between the passive and active spectroscopies.

In addition to the line brightness and $Z_{\text {eff }}$, a final comparison is made with the simulated total radiation due to nitrogen. Figure 7 shows that this follows closely the time evolution of the total radiation from the bulk plasma measured by bolometry. There is agreement in the absolute measurement to within a factor of two to three. In this nitrogen puffing discharge, the components of radiated power due to the different impurities were determined by an empirical method [14] which involves weighting individual line intensities and matching their sum to fit the total bolometric measurement of the radiation power. This method showed that about 


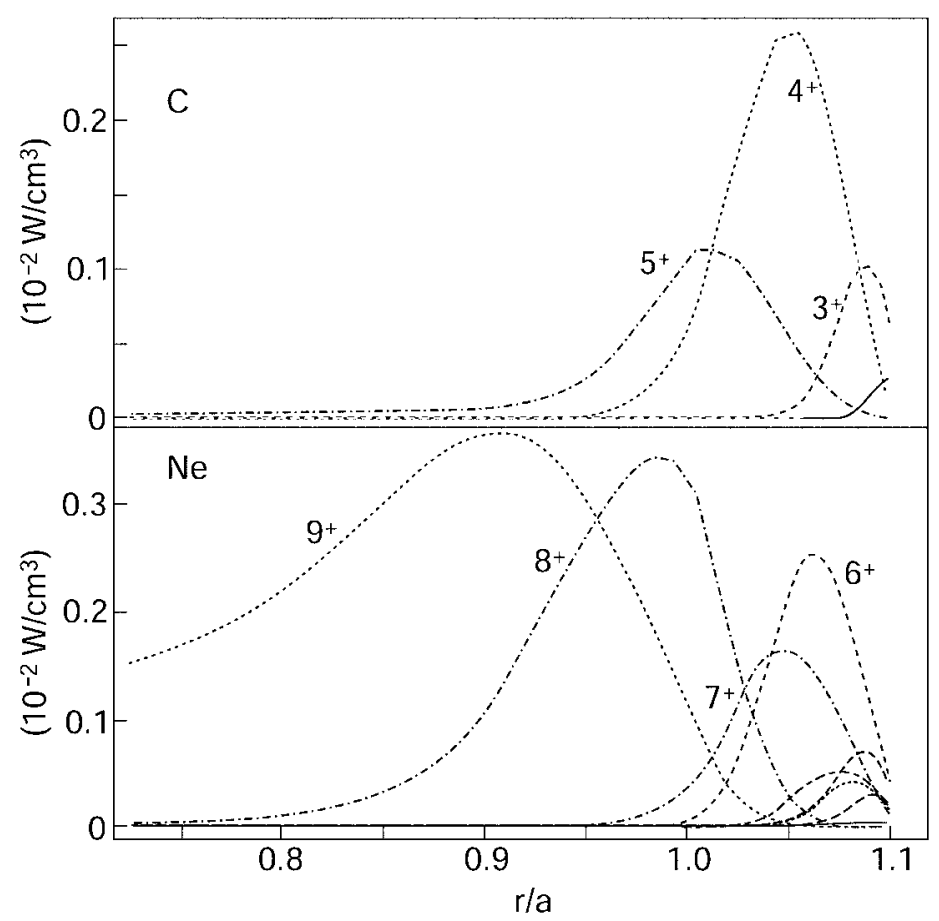

Figure 8. Simulated emission shells for carbon and neon in pulse 32385. (No puffing.)

$90 \%$ of the radiation in the bulk plasma was due to nitrogen. This demonstrates reasonable agreement between the simulated radiation and measurements from the bolometry diagnostics, with the simulation relying only on the spectroscopic measurements.

The consistency between the passive and active spectroscopies indicates a promising technique to deduce the impurity concentration through reliable transport simulations from passive spectroscopy that observes mainly the line radiation of impurities from the bulk plasma.

\section{The impurity radiation and contamination}

In the coronal ionization equilibrium, the calculated impurity cooling rates [15] show that the radiation from $\mathrm{C}, \mathrm{N}$ and $\mathrm{Ne}$ peak at electron temperatures of about 8,10 and $40 \mathrm{eV}$, respectively. In the JET plasma, the radiation tends to peak at higher temperatures because of transport effects.

Through satisfactory impurity transport simulations, non-coronal ionization equilibrium has been calculated and the simulated emission shells for carbon and neon (pulse 32385) are shown in figure 8 and those for nitrogen and carbon (pulse 33204) in figure 9. It can clearly be seen that there is significant neon emission from shells lying inside the innermost carbon shell, the neon emission corresponding to temperatures of up to about $1 \mathrm{keV}$, whereas the nitrogen emission profiles are close to those of carbon.

This behaviour is confirmed by the total radiation profiles for the three elements (figure 10(a)), which shows a comparison to the inversion of bolometry measurements in similar discharges (figure 10(b)) [16]. The radiation profiles and the simulations have a similar pattern.

The strong nitrogen puffing in pulse 33204 resulted in this element being the dominant 


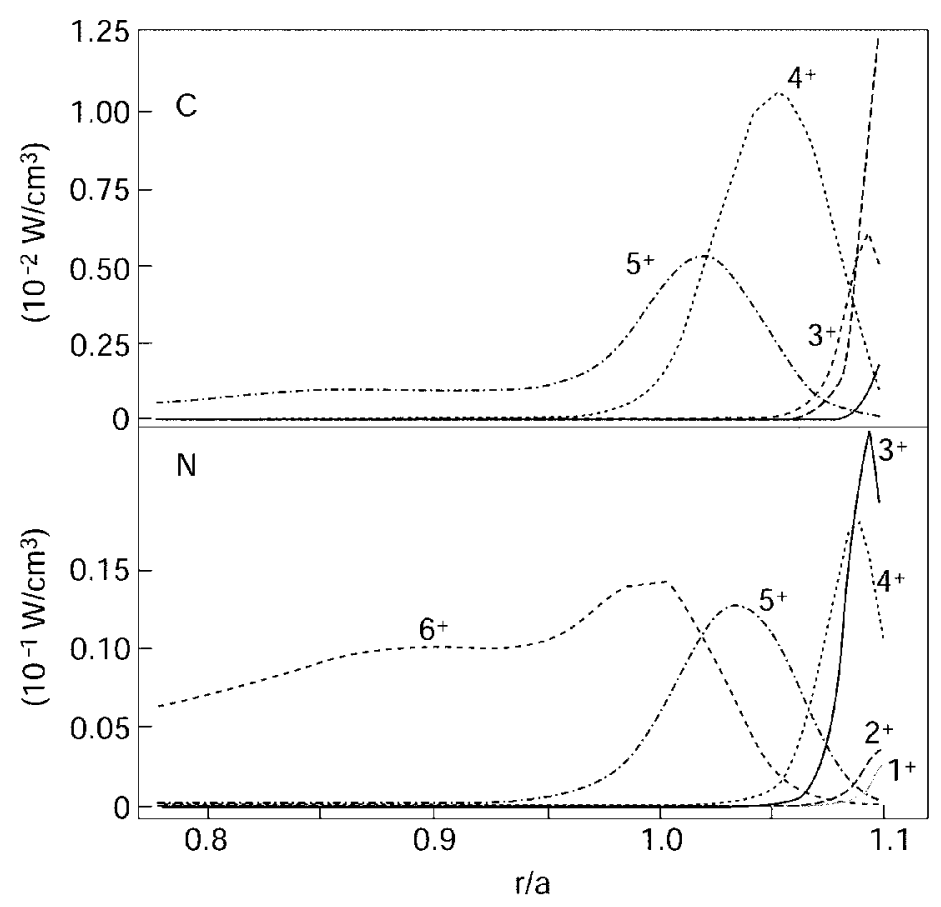

Figure 9. Simulated emission shells for carbon and nitrogen in pulse 33204. (No puffing.)

impurity in the bulk plasma. The component of $Z_{\text {eff }}$ due to nitrogen is therefore expected to be the largest contribution to the total $Z_{\text {eff }}$. The simulated $Z_{\text {eff }}$ profile is also consistent with the line-averaged $Z_{\text {eff }}$ of 3.0-3.4 derived from the bremsstrahlung measured by the visible spectrometer. This analysis shows that there is a radiating layer near the boundary of the plasma which has been established with the light impurities. In particular, using nitrogen as the radiator has better efficiency since its peak emissivity layer is further out.

The nitrogen concentration in the plasma centre is about $5 \%$ of electron density, rising to about $8 \%$ at $r / a=0.8$, the profiles clearly being hollow. In the neon puffing discharge 32385 , the hollow neon density profiles also lead to a hollow $\Delta Z_{\text {eff }}$. In this case, the neon concentration varies from about $1.3 \%$ at the plasma centre to a peak of about $2.5 \%$ at $r / a=0.8$.

\section{Conclusions}

The light impurity behaviour in the JET bulk plasma during ELMy H-modes has been studied in discharges in which two light impurities, neon and nitrogen, were puffed into the plasma. Prior to the introduction of these impurities the dominant impurity in these plasmas was the intrinsic carbon. In the impurity transport study, transport coefficients have been established which reproduce well the experiment data for ELMy H-modes. These transport coefficients are very similar for the injected neon and the intrinsic carbon impurities. The convection velocity appears to be directed inwards in the outer layers of the plasma column $(r / a>0.8)$ and outwards at inner radii $(r / a<0.7)$; the diffusivity is lower than $0.3 \mathrm{~m}^{2} \mathrm{~s}^{-1}$. The convection dominates the transport. The difference between the simulated transport coefficients and neoclassical theory predictions is smaller (within a factor of 2 to 10) in the core than it is in the edge region (with a factor of about 30 to 100). 


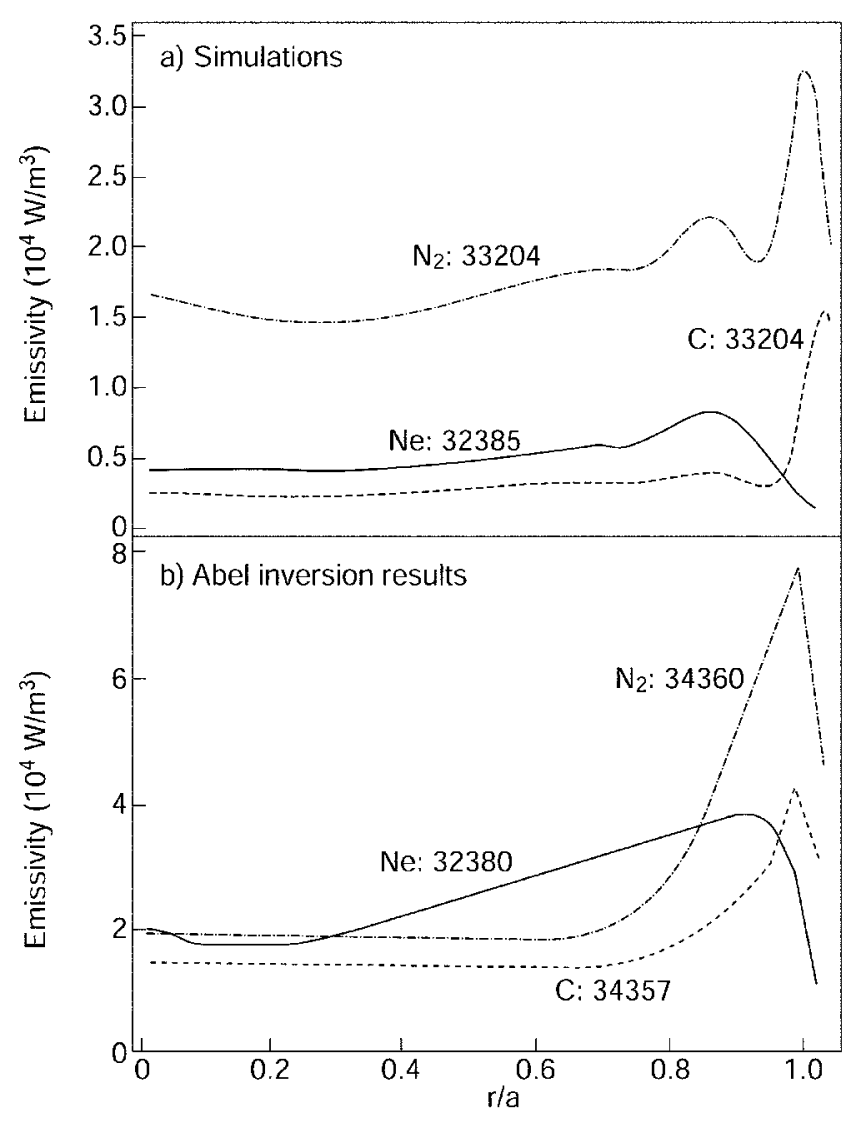

Figure 10. Simulated radiation profiles for $\mathrm{C}, \mathrm{Ne}$ and $\mathrm{N}$ (a). Radiation profiles for $\mathrm{C}, \mathrm{Ne}$ and $\mathrm{N}$ in similar discharges by Abel inversion methods (b).

Our simulations show that the measurements from the CXS measurements agree to better than a factor of two with those from an absolutely calibrated soft x-ray spectrometer observing passive emission. The radiation power profiles obtained in the simulations agree with those measured experimentally by bolometry and the computed elemental contributions to the total radiated power also agree with those obtained with empirical methods.

The neon radiation profile in the bulk plasma peaks at a radius inside that of nitrogen and carbon. In pulses where the injected gas dominates the impurities and therefore is the main contributor to the $Z_{\text {eff }}$, the $Z_{\text {eff }}$ profile appears to be hollow. Since the transport of the intrinsic light impurities is seen to be very similar to that of the injected neon and nitrogen, a hollow $Z_{\text {eff }}$ profile is expected to be a general feature of these ELMy H-mode discharges.

\section{Acknowledgments}

We would like to acknowledge the collaboration with the JET Experimental the UKAEA Task Agreement group. This work was funded by the UK Department of Trade and Industry and EURATOM. The completion of the paper was partly supported (H Chen) under the auspices of the US DOE by the University of California Lawrence Livermore National Laboratory under contract No W-7405-Eng-48. 


\section{References}

[1] Samm U et al 1993 Plasma Physics and Controlled Nuclear Fusion Research 1992 vol 1 (Vienna: IAEA) p 309

[2] Stambaugh R et al 1999 Nucl. Fusion 392391

[3] von Hellerman M and Summers H 1993 Atomic and plasma material interaction Processes in Controlled Thermonuclear Fusion ed R K Janev and H W Drawin (Amsterdam: Elsevier) pp 87-117

[4] Burnsley R 1992 Rev. Sci. Instrum. 635023

[5] Fonck R et al 1982 Appl. Opt. 212115

[6] Mast T et al 1985 Rev. Sci. Instrum. 56969

[7] Behringer K H 1997 Description of the impurity transport code STRAHL Report JET-R(1987)08

[8] Ginnella R et al 1994 Nucl. Fusion 341185

[9] Lauro-Taroni L et al 1996 Analisi del trasporto di impurezze nel JET unpublished presentation

[10] Summers H 1994 ADAS manual JET Report IR(12)

[11] Yushmanov P N et al 1990 Nucl. Fusion 301999

[12] Lauro-Taroni L et al 1994 Proc. 21st EPS (Montpellier, France) vol 1, p 102

[13] Barnsley R et al 1995 Proc. 22nd EPS Conf. (Bournemouth, UK) p 32

[14] Peacock N J et al 1999 Rev. Sci. Instrum. 70317

[15] Post D E et al 1977 At. Data Nucl. Data Tables 20397

[16] Reichle R et al 1995 Proc. 22nd EPS Conf. (Bournemouth, UK) p 305 